Sharif University of Technology
Scientia Iranica
SCIENTIA
IRAN ICAA
http://scientiairanica.sharif.edu

\title{
Non-linear seismic ground response analysis considering two-dimensional topographic irregularities
}

\author{
N. Soltani* and M.H. Bagheripour \\ Department of Civil Engineering, Faculty of Engineering, Shahid Bahonar University of Kerman, Kerman, Iran.
}

Received 5 June 2016; received in revised form 17 September 2016; accepted 10 December 2016

\section{KEYWORDS}

Topographic

irregularity;

FEM;

Viscous boundary;

Non-linear method.

\begin{abstract}
In the occurrence of an earthquake, local site conditions such as soil characteristics, dimension of topographic irregularities, seismic bedrock depth, etc. as well as characteristics of incident wave have important effects on seismic ground response. In this study, Finite Element Method (FEM) coupled with viscous boundaries is used to evaluate the effect of empty two-dimensional valleys on amplification or attenuation of seismic waves. Parametric studies are carried out and the effects of dimension of the topography, frequency of the incident wave, and bedrock depth on the seismic ground response are considered using non-linear method in a time domain analysis. Results are shown by means of horizontal and vertical amplification ratios in valley span and its surrounding area. It is concluded that displacement variation on ground surface due to topographical effects is a considerable factor to select a site location or design structures in the valley mount and its surrounding area.

(C) 2018 Sharif University of Technology. All rights reserved.
\end{abstract}

\section{Introduction}

Evaluation of surface ground motion due to seismic excitation at bedrock is one of the most important issues in geotechnical earthquake engineering. In common seismic events, body waves travel from the source mostly across a bedrock and finally end in soil layers. This is while most of the changes in the characteristics of ground motions occur in the soil layers [1]. These changes are generally studied with focusing and scattering of the phenomenon of seismic waves. Due to the drastic variation of the nature of seismic waves passing through soil layers, it is very important to incorporate realistic and precise seismic excitation models into the analysis of structural

\footnotetext{
*. Corresponding author.

E-mail addresses: n.soltani@eng.uk.ac.ir (N. Soltani); bagheri@uk.ac.ir (M.H. Bagheripour)
}

seismic response. Hence, vulnerability of structures can be a function of an important factor known as the site seismic response. Ground response analyses can properly satisfy the needs for realistic and precise seismic excitation in analysis of structures or soilstructure interaction.

Site effects are generally divided into two categories: effects of local deposits and those of topography. In this regard, the effect of topographic irregularities on ground motions is of great importance. Recent studies have indicated that topographic irregularities (e.g., mountain ridges or valley notches) cause significant changes to strong ground motions during earthquakes. Investigations into many earthquakes in the past have also indicated the effect of surface topographic changes on ground response. Evidences of topographic effects are available in Alaska 1964 [2], Canal Beagle Chile 1985 [3,4], Northridge 1994 [5,6], Athens 1999 [7], Umbria-Marche 1997 [8], and many others. The significance of local topographic irregularities is shown 
by the fact that earthquake causes vast damages to some certain regions and only slight damages to others.

Generally, numerical studies of the effect of topographic irregularities on the ground response are multidimensional analyses, which use various approaches such as Finite Element Method (FEM) (e.g., [9,10]); Boundary Element Method (BEM) (e.g., [11,12]); Spectral Element Method (SEM) (e.g., [13]); Finite Difference Method (FDM) (e.g., [14]); and hybrid methods such as coupled Finite and Infinite Element method (FE-IFE) (e.g., [15,16]), and coupled Finite and Boundary Element Method (FEM-BEM) (e.g., $[17,18])$. Detailed explanation of multidimensional analyses and comparison of results may be found in some references (e.g., $[9,19,20])$.

Extensive researches have been conducted to optimize ground seismic response analysis (e.g., [21-29]). For example, Lermo and Chávez-García [30] referred to the limitations of the classic method of spectral analysis, especially limitations imposed on field operation and the process of recording data. They also proposed a new method to study site effects. Following well-known Nakamura method, they used the ratio of the spectral amplitude of the horizontal to the vertical component of minor earthquakes. They also compared their results with those of the classic spectral approach. LeBrun et al. [31] carried out an experimental study on the topographic effects of a large hill with a height of $700 \mathrm{~m}$, width of $3 \mathrm{~km}$, and length of up to $6 \mathrm{~km}$ on seismic analysis. In the course of investigations, 7 seismographs were installed on a hill and a total of 58 earthquake records were obtained. They studied the ground motion with different methods: the Classical Spectral Ratios (CSR) and the horizontal to vertical spectral ratios were calculated both based on noise, so called Nakamura's method (HVNR), and then based on earthquake data, so called Receiver Function technique (RF). The comparison between these two methods showed that the $\mathrm{H} / \mathrm{V}$ method was able to suggest the fundamental frequencies of a hill. $\mathrm{Fu}$ [32] investigated effect of scattering surface waves by comparison of different theories. He also examined these waves by study of the propagation of $\mathrm{SH}$ waves through twodimensional models. He compared accuracy of different theories according to incident wavelength and dimension of model. Bouckovalas and Papadimitriou [14] analyzed the effect of topography on seismic waves using the Finite Difference Method (FDM). Their study was based on a site with uniform slope with a viscoelastic soil medium. The study was also conducted on the effects of different parameters on seismic ground motion, which was based on the vertical propagation of SV waves. Kamalian et al. [17] studied the effects of topography on a medium with heterogeneous materials. They used two-dimensional modeling method based on FEM-BEM. They also modeled distant boundaries using confining elements. They showed that their proposed method needed smaller time step than the BEM scheme. They also discussed the effective dimension of irregularities on seismic ground motion. Gatmiri et al. [18] investigated the effect of alluvial valleys on the amplification or attenuation of seismic waves. They also used a coupled model of FEM-BEM in which nearby field was modeled using FEM while the far field was modeled using BEM. They verified the accuracy of their method by numerical study and discussed that artificial waves developed at the truncation points of the model would vanish easier if an optimized method was used. Asgari and Bagheripour [33] performed a non-linear one-dimensional analysis of ground response using Hybrid Frequency-Time Domain (HFTD) approach. They used the advantages of both the time domain and the frequency domain methods to optimize the solution procedure. They showed the accuracy of their method by different illustrative examples. Di Fiore [10] considered the effect of incident wave frequency and gradient of slopes as a form of topographic irregularities on the amplification or attenuation of seismic waves using FEM. Investigations showed that amplification of seismic wave increased with increase in the gradient of slope. Also, analysis of 0.5 to $32 \mathrm{~Hz}$ incident wave frequencies revealed that the largest amplification of seismic waves was seen in frequencies between 4 to $12 \mathrm{~Hz}$. Bazrafshan Moghaddam and Bagheripour [34] proposed a new method for non-linear analysis of ground response using HFTD approach. This method was based on a non-recursive process and used a matrix-notation. Comparison of the results of the proposed method and those obtained by SHAKE and NERA software using records collected on the sites of several actual earthquakes showed accuracy and efficiency of the proposed method. Tripe et al. [35] conducted a study, using time domain approach, into the contribution of slopes of homogeneous and linearelastic soils to the amplification or attenuation of seismic waves using FEM.

One of the important problems in ground response analysis is the limitation or inability of numerical methods to simulate infinite boundaries and develop mathematical models for the passage of seismic waves as well as reduction in reflected waves from the boundaries into the models developed for soil layer. Such a deficiency greatly influences the results.

It should be noted that one-dimensional ground response analysis methods are suitable for horizontal or gently sloping grounds and, perhaps, for soil profiles having a set of parallel layers. However, other problems such as slopes; non-linear ground surfaces; topographic irregularities; and presence of heavy and stiff structures, buried structures, and tunnels require two- or even three-dimensional analysis [1].

In the present study, non-linear time domain 
analysis is carried to investigate the effect of twodimensional valleys on the amplification or attenuation of seismic waves using PLAXIS, which is one of the most powerful and applicable pieces of software to simulate infinite or semi-infinite soil and rock medium and is based on FEM. Using this software, one may apply proper boundaries to the FE models to simulate the infinite extent of a soil medium and prevent reflecting of artificial waves to the model.

Results are presented in non-dimensional amplification diagrams for both horizontal and vertical directions. In this investigation, a realistic numerical simulation of the medium is conducted to avoid reflection of seismic waves at boundaries using special boundary conditions known as "Viscous Boundaries". In fact, these boundaries are coupled with the rest of model's area simulated by FEM.

Application of such boundaries proposed in this research develops a powerful FE tools, namely, FEIFE, which diminishes difficulties caused by coupling of infinite elements with finite elements. Such an approach also helps developing a more rational method and, hence, achieving more acceptable results [9].

Amongst different types of seismic sites, empty valleys have been given less attention than other topographic irregularities. However, these types of valleys are important in theoretical studies and engineering applications since they may have large numbers of inhabitants due to various life resources. In addition, some of the important structures like dams, bridges, and briefly many infrastructures have been built in these types of valleys. In the following, the theoretical approach adopted in this study is discussed.

\section{Proposed theoretical approach}

\subsection{Equation of motion in multidimensional analysis}

The basic equation of motion for a system affected by a dynamic load in a finite element approach can be derived as:

$$
[M]\{\ddot{u}\}+[C]\{\dot{u}\}+[K]\{u\}=\{f\},
$$

where $[M],[C]$, and $[K]$ are mass, damping, and stiffness matrices, respectively. Also, $\{\ddot{u}\},\{\dot{u}\},\{u\}$, and $\{f\}$ are acceleration, velocity, displacement, and force vectors, respectively, which vary with time. In the following, like in Yoshida's manner [20], matrix and vector notations are not used for simplicity.

Generally, in the engineering practice and especially in the case of ground response analysis, relative displacement is considered; thus [20]:

$$
u=u_{r}+I u_{b} .
$$

In Eq. (2), $u_{r}$ is the relative displacement vector related to the displacements of the base, $u_{b}$ is the displacement vector for the base, and $I$ is a vector whose components are unity when the directions of the degree of freedom and loading are the same. These components are reduced to zero when those directions are different. Substituting Eq. (2) into Eq. (1), one can be inferred that:

$$
M\left(\ddot{u}_{r}+I \ddot{u}_{b}\right)+C\left(\dot{u}_{r}+I \dot{u}_{b}\right)+K\left(u_{r}+I u_{b}\right)=0 .
$$

Since $u_{b}$ is a rigid displacement in the domain, we have:

$$
K u_{b}=0
$$

Considering movement of domain in the air, one can obtain:

$$
C \dot{u}_{b}=0 \text {. }
$$

Further, the following relation can be inferred using Eqs. (3) to (5):

$$
M \ddot{u}_{r}+C \dot{u}_{r}+K u_{r}=-M I \ddot{u}_{b} .
$$

The subscript $r$ is further omitted for simplicity in the following, assuming that $u$ indicates relative displacement. Mass matrix can be formed using two different formulations: consistent mass matrix, in which the same interpolation function is applied in its development, and lumped mass matrix, in which more simplified interpolation function is used. However, the latter is a diagonal matrix and is easier to apply in various problems [20]. Detailed discussion on mass matrix formulations can be found in various references (e.g., [36]). In this study, lumped mass matrix formulation is adopted.

\subsection{Evaluation of damping matrix}

In finite element formulation, damping matrix is often formulated as a Rayleigh damping using mass and stiffness matrices:

$$
C=\alpha M+\beta K
$$

In the above equation, $\alpha$ and $\beta$ are coefficients, which are obtained in the following. Such a formulation for damping ensures that when the first part of the above equation is dominant, more low-frequency vibrations are damped; conversely, if the second part is dominant, more high-frequency vibrations are damped [37].

Substitution of Eq. (7) into Eq. (6) leads to [20]:

$$
\ddot{u}+\left(\alpha+\beta \omega_{0}^{2}\right) \dot{u}+\omega_{0}^{2} \dot{u}=0 .
$$

Also, according to Eqs. (6) to (8), for Single-DegreeOf-Freedom (SDOF) system, it can be inferred that:

$$
\xi=\alpha / 2 \omega_{0}+\beta \omega_{0} / 2
$$


For multi-degree-of-freedom system, by separation from the group of the SDOF system, one may reach the following relation:

$$
\xi_{i}=\alpha / 2 \omega_{i}+\beta \omega_{i} / 2,
$$

where subscript $i$ shows the $i$ th mode parameters.

The important range of deformations in the engineering practices occurs in the low-frequency modes. Since there are only two parameters in the Rayleigh damping, only two conditions can be satisfied at maximum. When the damping ratios in the first and second modes are determined, two parameters are obtained as [20]:

$$
\begin{aligned}
& \alpha=2 \omega_{1} \omega_{2} \frac{\xi_{1} \omega_{2}-\xi_{2} \omega_{1}}{\omega_{2}^{2}-\omega_{1}^{2}}, \\
& \beta=2\left(\frac{\xi_{2} \omega_{2}-\xi_{1} \omega_{1}}{\omega_{2}^{2}-\omega_{1}^{2}}\right) .
\end{aligned}
$$

The stiffness part of Rayleigh damping relation is more important than the mass part; therefore, one can evaluate $\beta$ from the damping at the predominant period considering $\alpha=0$ [20].

\subsection{Numerical integration scheme}

Generally, in seismic ground response problems, methods are categorized into linear, equivalent linear, and non-linear with different soil behavior assumptions. Analyses by these methods can be carried out either in time or frequency domain. The equivalent linear procedure is among the frequency domain methods and most of the non-linear methods use time domain approach .

In the time domain analysis, equation of motion is solved by integrating intervals in a small amount of time, which is called step by step time integration method. In this method, time intervals should be small in order to accurately follow loading variation as well as to fully account for changes in materials properties.

Time integration schemes can be categorized into explicit and implicit approaches. Despite some limitations, explicit scheme is relatively simple to formulate. Implicit scheme is rather complicated; however, the process of calculation is more trustworthy and accurate. Among implicit schemes, Newmark method is often adopted.

In this study, in order to solve the equation of motion in time domain, displacement and velocity in time, $t+\Delta t$, can be derived using Newmark method as follows [37]:

$$
\begin{aligned}
& u^{t+\Delta t}=u^{t}+\dot{u}^{t} \Delta t+\left((1 / 2-a) \ddot{u}^{t}+a \ddot{u}^{t+\Delta t}\right) \Delta t^{2}, \\
& \dot{u}^{t+\Delta t}=\dot{u}^{t}+\left((1-b) \ddot{u}^{t}+b \ddot{u}^{t+\Delta t}\right) \Delta t,
\end{aligned}
$$

$$
u^{t+\Delta t}=u^{t}+\Delta u .
$$

In the above equations, $\Delta t$ is time step and coefficients $a$ and $b$ determine the accuracy of the numerical time integration. The applicable range for $a$ and $b$ to ensure a stable solution is as follows [37]:

$$
\begin{aligned}
& b \geq 0.5, \\
& a \geq 1 / 4(0.5+b)^{2} .
\end{aligned}
$$

Interested readers can find various recommended values for $a$ and $b$ in different references. However, common values for them in [20,37] as $a=0.3025$ and $b=0.60$ are adopted here.

Eq. (12) can also be rewritten as [37]:

$$
\begin{aligned}
& \ddot{u}^{t+\Delta t}=\lambda_{0} \Delta u-\lambda_{2} \dot{u}^{t}-\lambda_{3} \ddot{u}^{t}, \\
& \dot{u}^{t+\Delta t}=\dot{u}^{t}+\lambda_{6} \ddot{u}^{t}+\lambda_{7} \ddot{u}^{t+\Delta t},
\end{aligned}
$$

or:

$$
\begin{aligned}
& \ddot{u}^{t+\Delta t}=\lambda_{0} \Delta u-\lambda_{2} \dot{u}^{t}-\lambda_{3} \ddot{u}^{t}, \\
& \dot{u}^{t+\Delta t}=\lambda_{1} \Delta u-\lambda_{4} \dot{u}^{t}-\lambda_{5} \ddot{u}^{t},
\end{aligned}
$$

in which the coefficients $\lambda_{0}$ to $\lambda_{7}$ are introduced in the time step and in $a$ and $b$ integration coefficients.

Using the implicit scheme, Eq. (1) at $t+\Delta t$ is generally written as follows [37]:

$$
M \ddot{u}^{t+\Delta t}+C \dot{u}^{t+\Delta t}+K u^{t+\Delta t}=f^{t+\Delta t} .
$$

Using Eqs. (14) to (16) and further mathematical operations, then by simplification, one can obtain:

$$
\begin{gathered}
\left(\lambda_{0} M+\lambda_{1} C+K\right) \Delta u=F_{\mathrm{ext}}^{t+\Delta t}+M\left(\lambda_{2} \dot{u}^{t}+\lambda_{3} \ddot{u}^{t}\right) \\
+C\left(\lambda_{4} \dot{u}^{t}+\lambda_{5} \ddot{u}^{t}\right)-F_{\mathrm{int}}^{t} .
\end{gathered}
$$

According to Eq. (12), $\Delta u$ can be obtained and added to the earlier displacement $\left(u^{t}\right)$.

\subsection{Boundary conditions and element characteristics}

Among the methods employed for the analysis of ground response, FEM is one of the most powerful ones because of capability of simulating complicated geological and geotechnical conditions, which are the matter of concern in this paper. Nevertheless, accurate simulation of boundary conditions and radial attenuation of wave energy is of particular importance to FE dynamic analysis. Application of boundaries with any constraint may lead to so called "trap box" effect for seismic waves in the model and, hence, to fictitious results.

In static deformation analysis, the vertical boundaries of the mesh are often fictitious boundaries and, 
thus, they do not affect the deformation behavior of the environment to be modeled [37]. However, for dynamic analysis, boundaries have to be placed far enough, farther than to be the boundaries in static analysis. Further, introduction of boundaries at far distance virtually means large mesh required in $\mathrm{FE}$ model with excessive elements, which also entails extra calculation time and core memory. As the size of the divisions decreases, the influence of boundary conditions becomes a prime concern.

Since seismic ground response analysis domain is mainly of rectangular shape, it can be assumed that most of the waves traveling in horizontal direction are surface waves and their wavelengths are larger than body waves; thus, one can use wide and thin elements [20]. In addition, adoption of large elements for an FE model filters high-frequency components whose short wavelength cannot be simulated with nodal points with long intervals.

Considering the mechanism governing the propagation of seismic waves, it has been found that size of elements should be less than $1 / 5$ to $1 / 6$ of the wavelength corresponding to the highest frequency content of the input motion [20].

\subsubsection{Viscous boundaries}

When viscous boundaries are applied, equivalent dampers are used instead of commonly used boundary constraints. Such equivalent dampers absorb stresses induced on the boundary. It further means that such dampers act when stress waves are travelling outwards from the domain.

Components of absorbed normal and shear stresses, when an equivalent damper is introduced in $x$ direction, are defined as follows:

$$
\begin{gathered}
\sigma_{n}=-c_{1} \rho v_{p} \dot{u}_{x}, \\
\tau=-c_{2} \rho v_{s} \dot{u}_{y} .
\end{gathered}
$$

In the above equations, $\rho$ is the density of materials, $v_{p}$ and $v_{s}$ are respectively the velocity of the compressional and shear waves, and $c_{1}$ and $c_{2}$ are relaxation coefficients that are applied to the model to enhance the performance of the viscous boundaries. The interesting point is that if incident compressional waves reach the model's vertical boundaries, $c_{1}$ and $c_{2}$ coefficients are reduced to unity $\left(c_{1}=c_{2}=1\right)$. However, in presence of shear waves, the damping effect of viscous boundaries would not be adequate if coefficients $c_{1}$ and $c_{2}$ are neglected. In fact, the effect of these boundaries is increased directly with increase in $c_{2}$ value. Recent studies have shown that application of $c_{1}=1$ and $c_{2}=0.25$ would optimize the absorbing effect of these boundaries [37]. Fundamental formulation of these viscous boundaries is based on the procedure described in [38].

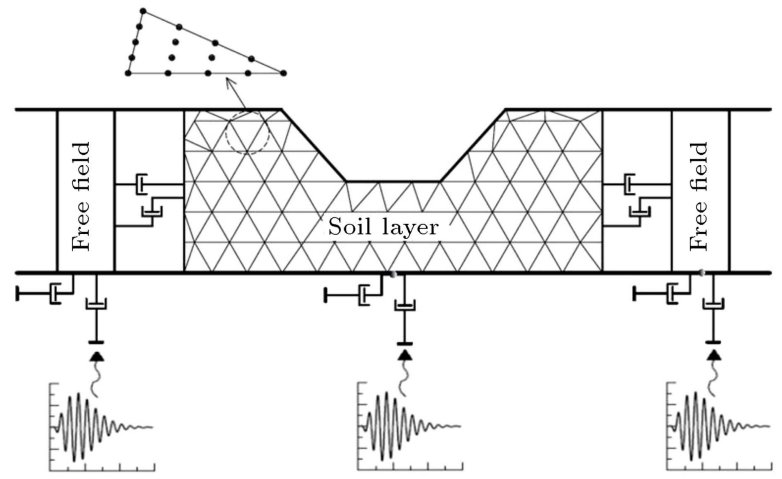

Figure 1. Schematic image of the model and 15-node element used in this research.

\section{Problem definition}

To investigate the effect of topographic irregularities and site effect parameters on seismic ground response, a valley environment is adopted as shown in Figure 1. To evaluate the amplification ratio due to interaction between topography and soil layer, a two-dimensional FEM approach is considered for parametric study. Soil layer is assumed overlain rigid bedrock and the adopted seismic excitation is an in-plane vertically propagating $\mathrm{SV}$ wave induced on bedrock. The obtained response is investigated at the ground level at various points for the effect of soil layer and topographic irregularities on amplification or attenuation of seismic waves.

15-node elements are used to model the soil medium because they provide more accurate results since they benefit from a better interpolation scheme (Figure 1). These elements have 2 degrees of freedom defined at every node and have 12 Gaussian points.

The accuracy of the proposed method is shown by comparison of the results obtained using the current method with those of FE-IFE method, including non-dimensional diagrams for horizontal and vertical displacement amplitudes, through the valley span and its surrounding area [9]. The satisfactory agreement between the results of two methods prove an acceptable performance of viscous boundaries in simulation of semi-infinite and infinite environments. Hence, the existing model is capable of simulating similar conditions.

\subsection{Frequency content of seismic excitation}

Two-dimensional site effects are important where the dimension of topography is approximately equal to the wavelength of the seismic wave [39]. In earthquake engineering, it has been shown that the frequency content of a strong earthquake almost ranges from 0.1 to $20 \mathrm{~Hz}$. Further, the velocity of seismic waves near the ground surface lies between 0.1 to $3 \mathrm{~km} / \mathrm{s}$; topographies having dimensions larger than tens of meters to several kilometers usually behave as in twodimensional site response models (e.g., $[1,10,40]$ ).

In order to facilitate study of the effect of the 
input harmonic wave's frequency content, a nondimensional parameter known as the dimensionless frequency, $a_{o}$, is defined based on the following relation:

$$
a_{o}=\frac{\omega h}{\pi V_{s}}
$$

where, $\omega$ is the angular frequency of the incident wave induced on the bedrock, $h$ is the maximum valley depth, and $V_{s}$ is the velocity of shear wave travelling through the soil medium.

To simplify evaluation of the impact of different parameters on seismic ground response analysis, results are shown in terms of amplification factor in horizontal and vertical directions as follows:

$$
\begin{aligned}
& \mathrm{HA}=u_{x} / u_{o}, \\
& \mathrm{VA}=u_{y} / u_{o},
\end{aligned}
$$

where, HA and VA are, respectively, the horizontal and vertical amplification. In the above equations, $u_{x}$ and $u_{y}$ denote displacement amplitude along $x$ and $y$ directions, respectively; also, $u_{o}$ is the amplitude of the incident wave. The incident wave amplitude is selected so that the maximum input acceleration (in depth) is equal to $0.35 \mathrm{~g}$. Diagrams of seismic amplification help in understanding the pattern of response along the valley span and its surrounding area.

\section{Parametric study and discussion}

As seen in Figure 2, the valley considered in this study is symmetric, whose maximum depth is $h$, while $H$ refers to depth of the bedrock. $L$ and $l$ are, respectively, halves of the top and bottom spans of valley and are adopted in this study by fixed values of $100 \mathrm{~m}$ and $50 \mathrm{~m}$.

Poisson's ratio is assumed to be constant and equal to 0.33 , while soil's modulus of elasticity is adopted as $2.4 \times 10^{7} \mathrm{kN} / \mathrm{m}^{2}$. The unit weight of soil is considered to be $23.54 \mathrm{kN} / \mathrm{m}^{3}$. The dimensions of the valley in real situation are basically within the studied range [15].

In this study, the effects of valley ratio $(h / H)$, frequency content of incident wave, and the depth of the bedrock on seismic ground response are considered. Generally, in this paper, because of the geometric symmetry and the vertically planar SV seismic excitation, the results for half of the valley are shown.

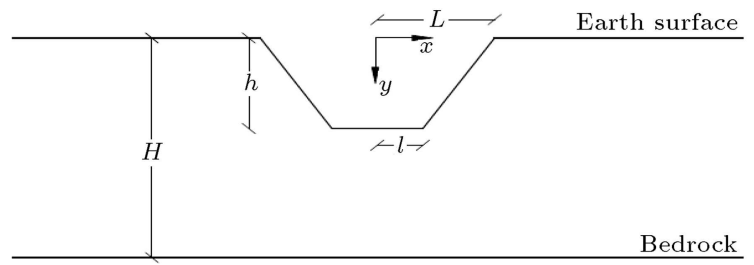

Figure 2. Configuration of the adopted valley.

\subsection{Effect of valley ratio}

In order to evaluate the effect of valley ratio, constant values of $h / H=0,1 / 4,2 / 4$, and $3 / 4$ are adopted. In this part, the depth of the bedrock is 200 meters.

Figure 3(a) shows that in the case of free field, amplification of the horizontal component nearly doubles, which reflects the extent of outcrop motion to the corresponding bedrock. In other shape ratios, as the distance from the valley center increases, the conditions of free field are also observed. Therefore, ground surface motions are always amplified compared with other points at depths.

As the parameter $h / H$ increases, amplification of the horizontal component of displacement in the center of the valley is increased. However, in all cases, except for the free field condition, which has a uniform amplification, the locus of maximum HA occurs in the valley center. Increasing $h / H$ causes $H A$ to reach a double quantity in the farther distances with respect to the center. It is important to note that the area affected by topographical problem varies depending on topographic dimensions. Therefore, model dimensions

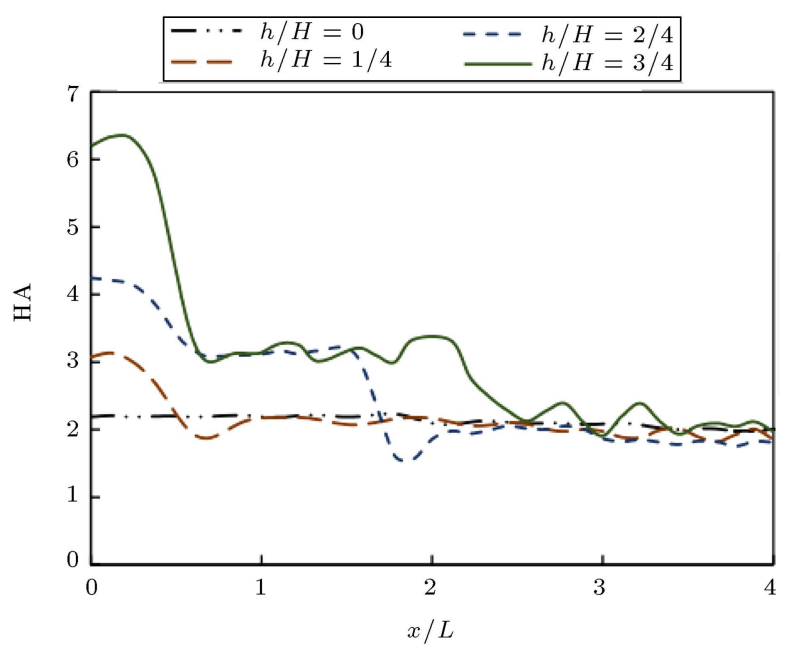

(a)

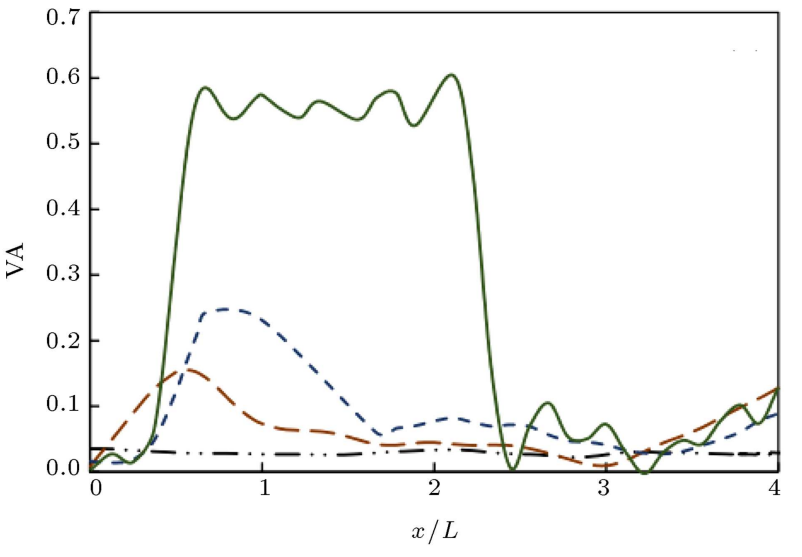

(b)

Figure 3. Seismic amplification comparison for different valley shape ratios $\left(a_{o}=1\right)$. 
are determined based on topographic dimensions. It further implies that by increase in the area of the valley, free field condition is attained in a farther distance.

According to Figure 3(b), although the incident wave is considered to be an SV wave propagating in vertical direction, the vertical component of the output wave at the ground surface does not vanish even in the case of free field condition. However, in the case of topographic irregularities, VA is larger than the same variable in free field condition. This phenomenon can be attributed to the interference of incident waves and their consecutive reflections in soil medium. At the valley center, due to the assumed symmetry of the valley and the upward propagation of incident wave, VA is virtually reduced to zero.

Increasing $h / H$ causes the maximum VA to increase correspondingly. As seen in Figure 3(b), the maximum occurs in $h / H=3 / 4$ and it continues to a relatively large distance from the edge of the valley. However, the locus of the maximum shifts from the body to top of the valley and it is sustained over farther distances as the depth of the valley increases. As well as HA, increasing $h / H$ causes the surrounding area to undergo more amplification.

It is found that increase in the depth of the valley not only increases the maximum values of $\mathrm{HA}$ and VA, but also sustains the amplification of the topography over farther distances. In other words, the effect of topographic irregularity on seismic ground motion becomes more considerable. Different behaviors with different depths of the valley can be attributed to 2 major factors: first, increase in valley's body slope, demonstrated by increase in depth while top and bottom spans of valley are fixed, causes increase in amplification. The reason behind such a phenomenon has been explained earlier by the current investigators [9] Second, increasing the valley depth and, consequently, an increase in its area cause more amplification by surface waves and their interference with incident and reflected waves.

\subsection{Effect of frequency content of input wave}

As mentioned earlier, frequency content of input wave is here referred to as $a_{o}$ parameter. In this study, constant values of $a_{o}=0.25,0.5$, and 1 are adopted for non-dimensional frequencies of seismic excitation and the shape ratio of the valley is considered as $h / H=1 / 2$ (where $h=100 \mathrm{~m}$ and $H=200 \mathrm{~m}$ ). The wavelengths of the incident waves are considered 1, 2, and 4 times the valley depth.

As can be inferred from Figure 4(a), HA increases in almost all parts of valley mount and its surrounding area as frequency content decreases. However, in frequency ratios of $a_{o}=0.25$ and 0.5 , the locus of maximum takes place at the top of the valley, but in $a_{o}=1$, it happens at its bottom. In other words, frequency content of the incident wave is one of the effective parameters to determine the locus of maximum HA. Decreasing the frequency content causes more oscillation in $\mathrm{HA}$ and this component reaches a value double the input wave in the farther distances from the center.

According to Figure 4(b), maximum VA occurs at top of the valley in different frequencies. Decreasing the frequency causes VA at farther distances from the valley center to reach a minimal value.

It should be noted that in addition to the surface topographical and mechanical properties of soil layer, different frequency contents of the incident wave due to change in $a_{o}$ are also effective in determination of model dimensions to attain free field condition. However, this frequency content also affects the quantities and loci of maximum and minimum HAs and VAs. It further implies that for sites having the same topographical but different seismological conditions, numerically simulated model may not have the same dimensions, since different frequency contents of seismic input wave

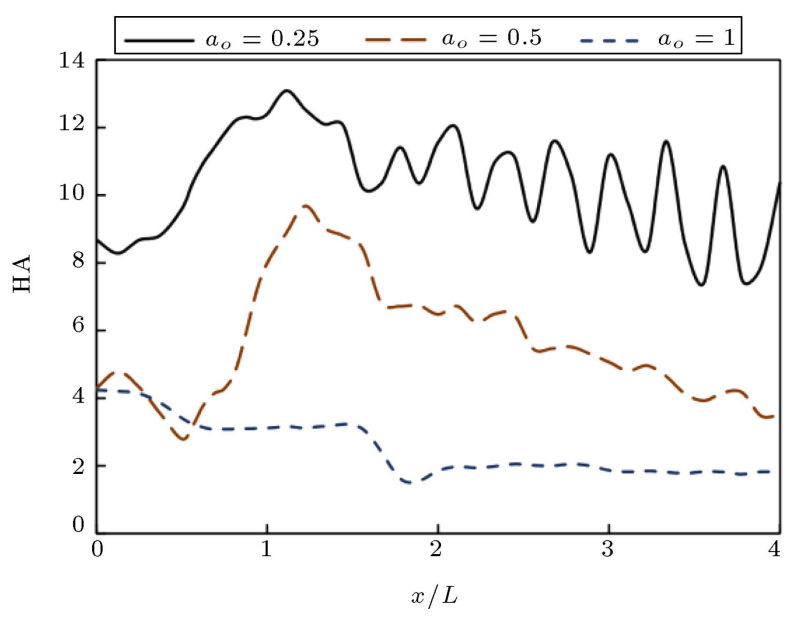

(a)

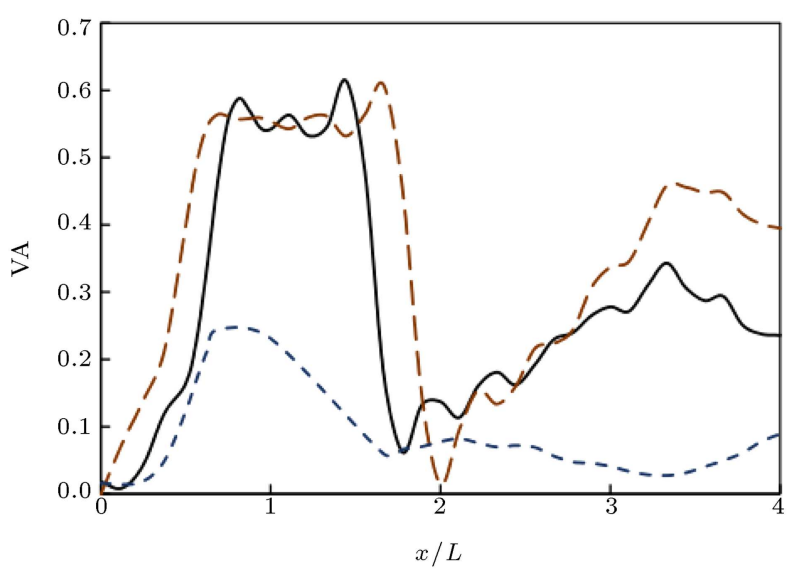

(b)

Figure 4. Seismic amplification comparison for different wave characteristics $(h / H=1 / 2)$. 
necessitate different extensions of the model to reach free field condition.

\subsection{Effect of bedrock depth}

In order to study the effect of bedrock depth, dimension of topography is kept constant while various ratios for $H / h$, namely, $1.5,2,2.5,3$, and 3.5 , are adopted. In this part, the height of valley is considered constant and equal to $100 \mathrm{~m}$.

According to Figure 5(a), it can be inferred that an increase in the bedrock depth causes HA to decrease in the bottom of the valley; however, it does not vary in the top of the valley. This phenomenon can be attributed to sharp angle at the bottom of topography, which may cause the waves to be trapped and reflected in multiple manners. This effect vanishes by increasing the depth of the bedrock. It is also important to note that the effect of topographic dimension on the ground seismic response is not considered as an absolute impact. In fact, it is rational to evaluate topographical dimension in relation to the bedrock

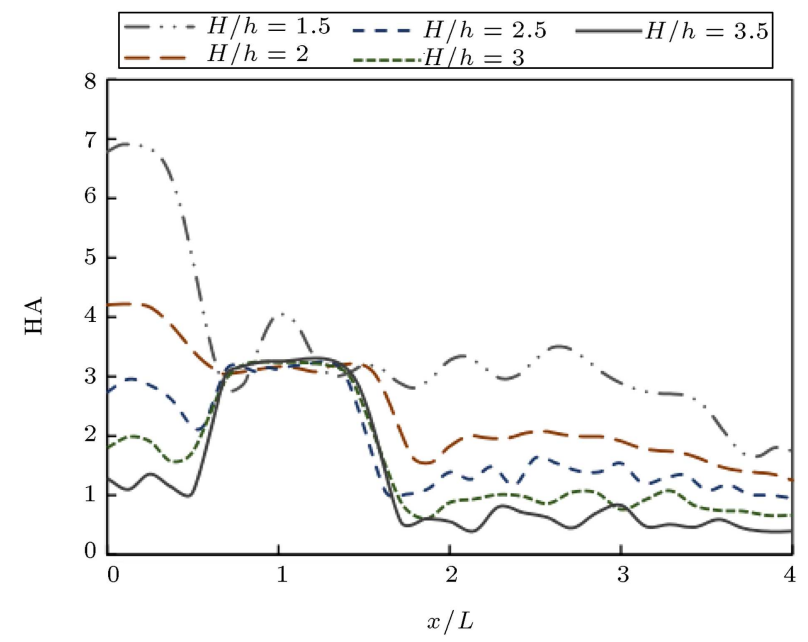

(a)

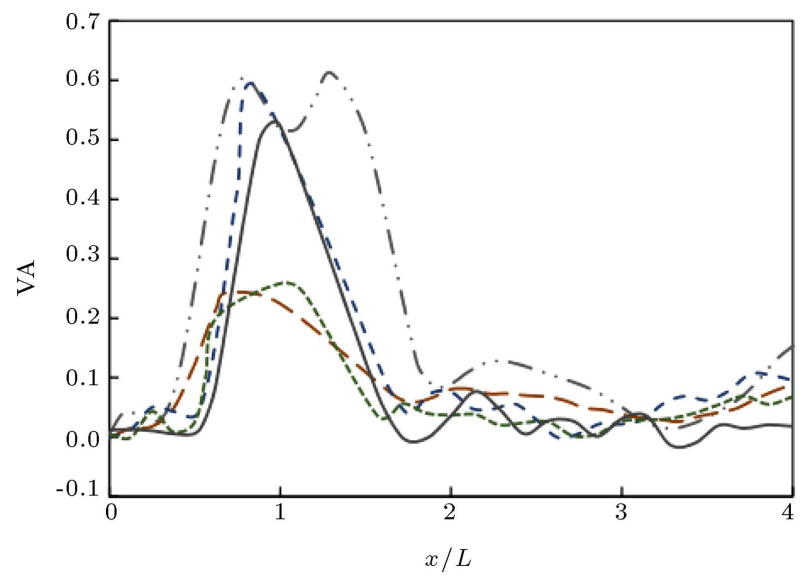

(b)

Figure 5. Seismic amplification comparison for different bedrock depths $\left(a_{o}=1\right)$. depth, since the ratio of the topographical dimension to the bedrock depth is important. Generally, in a particular topographical environment, increase in bedrock depth significantly reduces the effect of topographical conditions on the ground response.

Investigation into Figures 5(a) reveals that when the bedrock depth becomes more than double the valley height, the locus of maximum HA moves to the top of the valley; however, in other cases, it would be at the bottom of the valley.

It is noted that variation in soil layer thickness results in alternation of natural frequency of the soil layer, which, in turn, induces a significant impact on the HA component of the response, particularly at the bottom of the valley. The effect of this parameter is very evident on the VA as will be discussed in the following.

Evaluation of Figure 5(b) shows that in all bedrock depths considered here, the locus of maximum VA occurs at the top of the valley. However, at $H / h=1.5,2.5$, and 3.5 , its value is larger than other ratios considered.

Since the natural frequency of the soil layer depends mostly on the shear wave velocity and the thickness of the soil layer, it is seen that in $H / h=$ $1.5,2.5$, and 3.5, the natural frequency of the soil mass occurs almost close to the frequency of harmonic incident load and, as a result, resonance occurs. This phenomenon causes the maximum amplitudes to reach the highest value in these 3 states. It is noteworthy that because the wave energy dissipation is considered as the damping ratio in the model, the amplifications reach a local maximum, but never attain an infinite value.

In order to investigate the sensitivity of soil mass variation (due to change in bedrock depth), various values for the $H / h$ ratio in the range of $1.5<H / h<$ 2 are considered for the simulated model. For this purpose, the $H / h$ values of $1.5,1.7,1.8,1.9$, and 2 are adopted and compared upon the results reached. As can be inferred from Figure 6, increasing the bedrock depth causes HA and VA to decrease from the value corresponding to the first natural frequency.

According to the results, variations of displacement are seen inside the valley as well as in the surrounding environment. Displacement variations are caused by surface waves and their interference with incident and reflected waves. Consequently, such variations can have a significant effect on the seismic response of structures, which may be constructed in the valley and its surrounding area . Therefore, seismic design in such environments requires complementary investigations.

It is deduced from the discussion given in preceding sections that topographical effect on seismic ground response may directly affect the selection of appropriate 


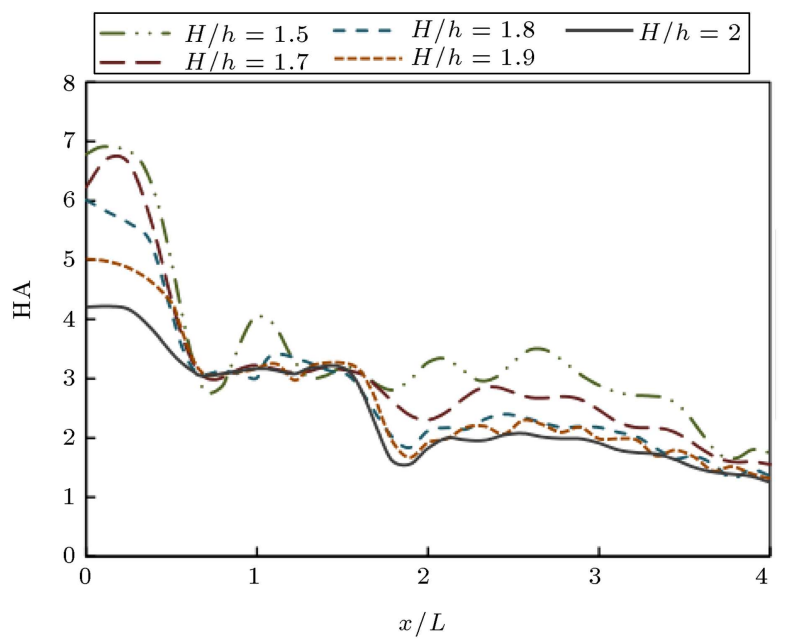

(a)

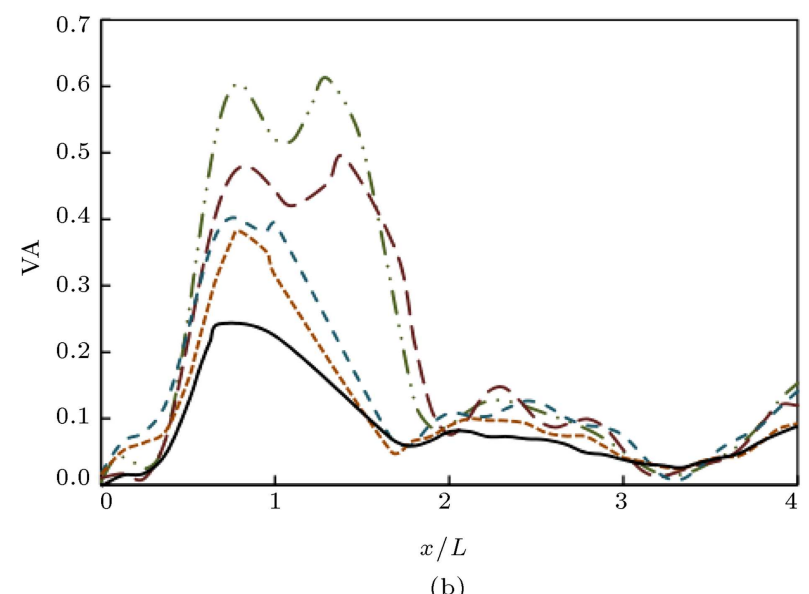

Figure 6. Seismic amplification comparison for sensitivities of bedrock depths $\left(a_{o}=1\right)$.

site locations for important projects such as dams, pipelines, etc. It further implies that comprehensive studies, especially with regards to topographical impact on seismic response, are required to optimize the safety factor and, hence, reduce the seismic risk and the cost of projects. Evaluation of the results also reveals that, for a structure built inside and around steeper valley banks, stronger $\mathrm{HA}$ and VA should be considered, irrespective of the fact that incident earthquake wave only has the horizontal acceleration component.

\section{Conclusion}

In this study, the seismic response of a site was considered regarding topographical irregularities. Horizontal and vertical amplification factors due to twodimensional topographic effect were evaluated using a realistic model based on FEM coupled with viscous boundaries. The solution was based on fully non-linear soil behavior in time domain. Using the numerical approach allowed a parametric study of effects of valley ratio, frequency of incident wave, and bedrock depth on $\mathrm{HA}$ and VA.

Basic conclusions drawn from the numerical simulation are as follow:

- The loci of maximum and minimum HA and VA depend not only on the geometric characteristics of the site, but also on the frequency content of the incident wave. It is shown that different sites with the same geometric characteristics may require different seismic ground responses and special considerations according to seismic zone of the site;

- It is also concluded that the severity of the effects of the incident wave frequency on seismic response are controlled by the geometric characteristics of the site;

- Dimension of the mesh domain in numerical studies in seismic ground response analysis depends on both geometric characteristics and seismic zone of the interested site;

- To obtain an optimized model size and to reach free field condition, dimension of the valley should not be regarded as a unique parameter. Rather, these dimensions should be normalized with respect to bedrock depth;

- It is concluded that displacement variation on ground surface due to topographical effects is an important parameter to select the site location or design of important structures, especially those with linear behavior.

\section{References}

1. Kramer, S.L. "Geotechnical earthquake engineering", In Prentice-Hall International Series in Civil Engineering and Engineering Mechanics, Prentice-Hall, New Jersey (1996).

2. Idriss, I. "Finite element analysis for the seismic response of earth banks", Journal of Soil Mechanics \& Foundations Div, 94, pp. 617-636 (1968).

3. Celebi, M. "Topographical and geological amplifications determined from strong-motion and aftershock records of the 3 March 1985 Chile earthquake", Bull. Seismol. Soc. Am., 77, pp. 1147-1167 (1987).

4. Celebi, M. "Topographical and geological amplification: case studies and engineering implications", Struct. Saf., 10, pp. 199-217 (1991).

5. Bouchon, M. and Barker, J.S. "Seismic response of a hill: the example of Tarzana, California", Bull. Seismol. Soc. Am., 86, pp. 66-72 (1996).

6. Celebi, M. "Northridge (California) earthquake: unique ground motions and resulting spectral and site effects", International Conference on Seismic Zonation, pp. 988-995 (1996). 
7. Gazetas, G., Kallou, P., and Psarropoulos, P. "Topography and soil effects in the MS 5.9 Parnitha (Athens) earthquake: the case of Adames", Nat. Hazards., 27, pp. 133-169 (2002).

8. Marsan, P., Milana, G., Pugliese, A., and Sano', T. "Local amplification effects recorded by a local strong motion network during the 1997 UmbriaMarche Earthquake", Proc. 12th World Conference on Earthquake Engineering, Aukland New Zealand, Paper No. 1046 (2000).

9. Soltani, N. and Bagheripour, M.H. "Seismic wave scatter study in valleys using coupled $2 \mathrm{D}$ finite element approach and absorbing boundaries", Scientia Iranica, 24, pp. 110-120 (2017).

10. Di Fiore, V. "Seismic site amplification induced by topographic irregularity: Results of a numerical analysis on 2D synthetic models", Eng. Geol., 114, pp. 109-115 (2010).

11. Kham, M., Semblat, J.F., and Bouden-Romdhane, N. "Amplification of seismic ground motion in the Tunis basin: Numerical BEM simulations vs experimental evidences", Engineering Geology, 154, pp. 8086 (2013).

12. Nguyen, K.V. and Gatmiri, B. "Evaluation of seismic ground motion induced by topographic irregularity", Soil Dyn. Earthquake Eng., 27, pp. 183-188 (2007).

13. He, CH. H., Wang, J.T., Zhang, CH.H., and Jin, F. "Simulation of broadband seismic ground motions at dam canyons by using a deterministic numerical approach", Soil Dyn. Earthquake Eng., 76, pp. 136$144(2015)$.

14. Bouckovalas, G.D. and Papadimitriou, A.G. "Numerical evaluation of slope topography effects on seismic ground motion", Soil Dyn. Earthquake Eng., 25, pp. 547-558 (2005).

15. Zhao, C. and Valliappan, S. "Incident P and SV wave scattering effects under different canyon topographic and geological conditions", Int. J. Numer. Anal. Methods Geomech., 17, pp. 73-94 (1993).

16. Duzgun, O.A. and Budak, A. "A study on soilstructure interaction analysis in canyon-shaped topographies", Sadhana., Indian Academy of Sciences., 35, pp. 255-277 (2010).

17. Kamalian, M., Jafari, M.K., Sohrabi-bidar, A., Razmkhah, A., and Gatmiri, B. "Time-domain twodimensional site response analysis of non-homogeneous topographic structures by a hybrid BE/FE method", Soil Dyn. Earthquake Eng., 26, pp. 753-765 (2006).

18. Gatmiri, B., Arson, C., and Nguyen, K. "Seismic site effects by an optimized 2D BE/FE method I. Theory, numerical optimization and application to topographical irregularities", Soil Dyn. Earthquake Eng., 28, pp. 632-645 (2008).
19. Lo Presti, D., and Ferrini, M., Special Issue of the Italian Geotechnical Review: The Seismic Response Analysis at Castelnouvo Garfagnana (2002).

20. Yoshida, N., Seismic Ground Response Analysis, Springer (2015).

21. Bagheripour, M.H. and Marandi, S.M. "A Numerical model for unbounded soil domain in earthquake SSI analysis using periodic infinite elements", Int. J. Civ. Eng., 3, pp. 96-111 (2005).

22. Bagheripour, M.H., Rahgozar, R., and Malekinejad, M. "Efficient analysis of SSI problems using infinite elements and wavelet theory", Geomech. Eng., 2(4), pp. 229-252 (2010).

23. Nimtaj, A. and Bagheripour, M.H. "Non-linear seismic response analysis of the layered soil deposit using hybrid frequency-time domain (HFTD) approach", European Journal of Environmental and Civil Engineering, 17, pp. 1039-1056 (2013).

24. Kara, H.F. and Trifunac, M.D. "Two-dimensional earthquake vibrations in sedimentary basins-SH waves", Soil Dyn. Earthquake Eng., 63, pp. 69-82 (2014).

25. Ghaemian, M. and Sohrabi-Gilani, M. "Seismic responses of arch dams due to non-uniform ground motions", Scientia Iranica, 19, pp. 1431-1436 (2012).

26. Khanbabazadeh, H. and Iyisan, R. "A numerical study on the $2 \mathrm{D}$ behavior of the single and layered clayey basins", Bull Earthquake Eng., 12, pp. 1515-1536 (2014).

27. Javdanian, H., Jafarian, Y., and Haddad, A. "Predicting damping ratio of fine-grained soils using soft computing methodology", Arabian Journal of Geosciences, 8, pp. 3959-3969 (2015).

28. Jafarian, Y., Haddad, A., and Javdanian, H. "Predictive model for normalized shear modulus of cohesive soils", Acta Geodynamica et Geomaterialia, 11, pp. 89100 (2014).

29. Soltani, N. and Bagheripour, M.H. "Boundary simulation with dampers using finite element method in nonlinear seismic ground response analysis", Geodynamics Research International Bulletin, 3, pp. 16-26 (2015).

30. Lermo, J. and Chávez-García, F.J. "Site effect evaluation using spectral ratios with only one station", Bull. Seismol. Soc. Am., 83, pp. 1574-1594 (1993).

31. LeBrun, B., Hatzfeld, D., Bard, P., and Bouchon, M. "Experimental study of the ground motion on a large scale topographic hill at Kitherion (Greece)", $J$. Seismolog., 3, pp. 1-15 (1999).

32. Fu, L.Y. "Rough surface scattering: comparison of various approximation theories for $2 \mathrm{D}$ SH waves", Bull. Seismol. Soc. Am., 95, pp. 646-663 (2005). 
33. Asgari, A. and Bagheripour, M.H. "Earthquake response analysis of soil layers using HFTD approach", The GeoShanghai 2010 International Conference, Shanghai, China (2010).

34. Bazrafshan Moghaddam, A. and Bagheripour, M.H. "Ground response analysis using non-recursive matrix implementation of hybrid frequency-time domain (HFTD) approach", Scientia Iranica, 18, pp. 11881197 (2011).

35. Tripe, R., Kontoe, S., and Wong, T. "Slope topography effects on ground motion in the presence of deep soil layers", Soil Dyn. Earthquake Eng., 50, pp. 72-84 (2013).

36. Desai, C.S. and Kundu, T., Introductory Finite Element Method, CRC Press (2001).

37. Brinkgreve, R. "Plaxis: finite element code for soil and rock analyses: 2D-Version 8:[user's guide]", Balkema (2002).

38. Lysmer, J. and Kuhlemeyer, R.L. "Finite dynamic model for infinite media", Journal of Engineering Mechanics Division, 95, pp. 859-878 (1969).

39. Bouchon, M. "Effect of topography on surface motion", Bull. Seismol. Soc. Am., 63, pp. 615-632 (1973).

40. Pagliaroli, A., Lanzo, G., and D'Elia, B. "Numerical evaluation of topographic effects at the Nicastro ridge in Southern Italy", J. Earthquake Eng., 15, pp. 404432 (2011).

\section{Biographies}

Navid Soltani received his BS degree in Civil Engineering from Yazd University in 2007 and his MS degree in Geotechnical Engineering from Shahid Bahonar University of Kerman in 2010. In 2012, he became a PhD student at Shahid Bahonar University of Kerman. He has published 12 journal and conference papers as well as several research projects with different governmental and private organizations. His research interests include geotechnical earthquake engineering, especially seismic ground response analysis; soil-structure interaction; and numerical methods.

Mohammad Hossein Bagheripour received his BS degree in Civil Engineering from Shahid Bahonar University of Kerman in 1988. He worked as consulting engineer in civil projects in south-eastern Iran before moving to Australia to continue his postgraduate studies. In 1993, he received his MEng Degree from the University of Sydney and was also awarded a $\mathrm{PhD}$ degree in Geotechnical Engineering in 1997 for his continuing research work on jointed rock mechanics. Immediately after graduation, he returned to Kerman, Iran, where he is currently Professor in the Civil Engineering Department of Shahid Bahonar University of Kerman. His research interests include soil and rock mechanics in general, and earthquake geotechnical engineering in particular. He has published numerous papers in various international and national journals and presented many others at conferences. 